Canad. Math. Bull. Vol. 21 (2), 1978

\title{
ON A RESULT OF LEVITZKI
}

\author{
BY \\ B. FELZENSZWALB ${ }^{(1)}$
}

A well known result of Levitzki [2, Lemma 1.1] is the following:

TheOREM. Let $R$ be a ring and $U$ a non-zero one-sided ideal of $R$. Suppose that given $a \in U, a^{n}=0$ for a fixed integer $n \geq 1$; then $R$ has a non-zero nilpotent ideal.

The purpose of this note is to observe some additional results which are related to the above.

We being with

THEOREM 1. Let $R$ be a ring with no non-zero nil ideals and $U$ an ideal of $R$. Suppose that $a \in R$ is such that for every $x \in U, a x^{n(x)}=0$ where $n(x) \geq 1$ depends on $x$; then $a U=U a=0$.

Proof. Let $V=\left\{r \in R \mid r x^{n}=0, n=n(r, x) \geq 1\right.$, all $\left.x \in U\right\}$. Then $V$ is a left ideal of $R$ and $V \cap U$ is nil. We proceed now to show that $V^{2} U \subset V \cap U$.

Let $v_{1} \in V$ and $u \in U$. Then there exists $k \geq 1$ such that $v_{1}\left(u v_{1}\right)^{k}=0$. Hence, if $y=v_{1} u, y^{k+1}=0$ and, in particular $y$ is quasi-regular.

Let $v_{2} \in V$. If $x \in U, z=(1+y) x(1+y)^{-1} \in U$; so, there exists $n \geq 1$ such that $v_{2} z^{n}=0$. Since $x=(1+y)^{-1} z(1+y)$ we have that $(1+y)^{-1} v_{2}(1+y) x^{n}=0$. That is, $(1+y)^{-1} v_{2}(1+y) \in V$.

Thus, if $x \in U$

$$
\begin{gathered}
v_{2} x^{m}=0 \\
(1+y)^{-1} v_{2}(1+y) x^{m}=0
\end{gathered}
$$

holds for a suitable $m \geq 1$. It follows that $\left(v_{2} v_{1}\right) u x^{m}=v_{2} y x^{m}=v_{2}(1+y) x^{m}=0$; hence $\left(v_{2} v_{1}\right) u \in V$. In other words, we have shown that $V^{2} U \subset V \cap U$. Since $V \cap U$ is nil, by our assumptions on $R, V^{2} U=0$. But this gives $(V U)^{3}=$ $(U V)^{3}=0$ and consequently, since $R$ has no non-zero nilpotent ideals, $V U=$ $U V=0$. Since $a \in V$ the result follows.

It was shown in [1] that if $R$ is a ring with no non-zero nil right ideals and $a \in R$ is such that for every $x \in R, a x^{n} a=0$ where $n=n(x) \geq 1$ depends on $x$, then $a=0$. The idea used to prove this can also be used to prove the following

Received by the editors June 27, 1977 and in revised form, September 12, 1977. 
THEOREM 2. Let $R$ be a ring with no non-zero nil right ideals and $U$ a left (respectively right) ideal of $R$. Suppose that $a \in R$ is such that for every $x \in U$, $a x^{n(x)}=0$ where $n(x) \geq 1$ depends on $x$; then $a U=U a=0$ (respectively $\left.a U=0\right)$.

Proof. The situation when $U$ is a right ideal of $R$ follows immediately because in this case, if $x \in U, a(x a)^{k}=0$ for some $k \geq 1$; so $(a x)^{k+1}=0$. That is, $a U$ is a nil right ideal of $R$. By our assumptions on $R$ we must have $a U=0$.

Suppose now $U$ is a left ideal of $R$. Let $r \in U$ with $r^{2}=0$. Then, by hypothesis, there exists $m \geq 1$ such that $a(a r)^{m}=0$ and $a(r+a r)^{m}=0$. Hence, on expansion of the last equation we get $(a r)^{m}=0$. In other words, $a r$ is nilpotent for every $r \in U$ with $r^{2}=0$.

Let $V=\left\{y \in R \mid y x^{n}=0, n=n(y, x) \geq 1\right.$, all $\left.x \in U\right\}$. Then $V$ is a left ideal of $R$ and, by what we deduced before, $V r$ is nil for every $r \in U$ with $r^{2}=0$. Since $\boldsymbol{R}$ has no non-zero nil right ideals, and hence no non-zero nil left ideals, we obtain

$$
V r=0 \text { for every } r \in U \text { with } r^{2}=0 .
$$

Let $x \in U$. We claim that if $y \in V, y x$ is nilpotent. We go by induction on $n$ such that $y x^{n}=0$. If $n=1$ there is nothing to show. Suppose $n>1$; then $x y x^{n-1} \in U$ and $\left(x y x^{n-1}\right)^{2}=0$, so, by (1), $y x y x^{n-1}=0$. Since $y x y \in V$ our induction gives us $(y x)^{2}$ nilpotent; hence $y x$ is nilpotent.

So, $U y$ is nil for every $y \in V$. Since $R$ has no non-zero nil right ideals we get $U V=0$ and, a fortiori, $V U=0$. Since $a \in V$ this proves the theorem.

We note that if in Theorem 2 the integers $n(x)$ have a finite maximum as $x$ ranges over $U$ the conclusion remains valid if we replace the hypothesis "with no non-zero nil right ideals" by "with no non-zero nilpotent ideals" (the proof goes just the same with the use of Levitzki's result and with the induction hypothesis " $y x^{n}=0$ implies $\left.(y x)^{2^{n}}=0 "\right)$. This yields a generalization of Levitzki's Theorem.

The question of whether or not Theorem 2 remains valid if we replace the hypothesis "with no non-zero nil right ideals" by its two-sided version "with no non-zero nil ideals" is now readily seen to be equivalent to the Koethe conjecture: a nil one-sided ideal in a ring $R$ generates a two-sided nil ideal.

\section{REFERENCES}

1. B. Felzenszwalb, Rings Radical over Subrings, Israel J. Math. 23 (2) (1976) 156-164.

2. I. N. Herstein, Topics in Ring Theory, University of Chicago Press, 1969.

INSTITUTO DE MATEMÁTICA

UNIVERSIDADE FEDERAL do RIO DE JANEIRO

C.P. 1835 ZC-00

20.000 Rio DE JANEIRO, R. J., BRAZIL

(1) This work was supported by FINEP, Brazil. 